\title{
Heteromerization of TRP channel subunits: extending functional diversity
}

\author{
Wei Cheng ${ }^{1,2}$, Changsen Sun ${ }^{1}$, Jie Zheng ${ }^{2 凶}$ \\ ${ }^{1}$ Lab of Biomedical Optics, College of Physics and Optoelectronic Engineering, Dalian University of Technology, Dalian 116023, \\ China \\ 2 Department of Physiology and Membrane Biology, University of California School of Medicine, Davis CA 95616, USA \\ $\triangle$ Correspondence: jzheng@ucdavis.edu \\ Received August 16, 2010 Accepted September 1, 2010
}

\begin{abstract}
Transient receptor potential (TRP) channels are widely found throughout the animal kingdom. By serving as cellular sensors for a wide spectrum of physical and chemical stimuli, they play crucial physiological roles ranging from sensory transduction to cell cycle modulation. TRP channels are tetrameric protein complexes. While most TRP subunits can form functional homomeric channels, heteromerization of TRP channel subunits of either the same subfamily or different subfamilies has been widely observed. Heteromeric TRP channels exhibit many novel properties compared to their homomeric counterparts, indicating that co-assembly of TRP channel subunits has an important contribution to the diversity of TRP channel functions.
\end{abstract}

KEYWORDS co-assembly, molecular mechanism, diversification, nonselective cation channel, polymodal receptor, multi-subunit protein complex

\section{INTRODUCTION}

The first member of transient receptor potential (TRP) channels was identified in 1969 in Drosophila mutants that displayed a transient response to light (Cosens and Manning, 1969). In the following 40 years, not only Drosophila TRP channels received intense attention but also their homologs in other species were eagerly identified in realization of their involvement of a wide spectrum of cellular sensing functions. To date, 28 mammalian TRP channel genes are known. Additional TRP channel genes are found in other species such as worm, fruit fly and zebra fish. Indeed, the TRP channel superfamily has bloomed to more than 50 members of mostly nonselective cation ion channels. According to similarity in their primary sequences, TRP channels are classified into seven subfamilies: TRPC (canonical), TRPV (vanilloid), TRPM (melastatin), TRPP (polycystin), TRPML (mucolipin), TRPA (ankyrin) and TRPN (NO mechanopotential) (Clapham, 2003; Nilius, 2007b). As expected for such a large family of cation channels, TRP channels are widely found throughout the biologic kingdom from yeast and fly to mouse and human. They are expressed in both sensory neurons and non-sensory cells (Stowers et al., 2002; Zhang et al., 2003; Zhou et al., 2003).

Structurally, TRPs are tetrameric cation channels (GarcíaSanz et al., 2004). Each channel subunit contains six transmembrane segments (S1-S6) and a pore-forming region formed by a loop between the S5-S6 segments. Both the amino $(\mathrm{N})$ terminus and the carboxyl $(\mathrm{C})$ terminus are intracellularly located. Most of the TRP channels have an $\mathrm{N}$-terminal ankyrin repeat domain and a C-terminal TRP domain. The long $\mathrm{N}$ and $\mathrm{C}$ termini of TRPs also contain several additional regulatory domains which are relatively conserved in most of the TRPs (Fig. 1). For example, a coiledcoil domain present in TRPCs, TRPPs and TRPMs; TRPCs and TRPVs possess a PDZ-binding domain in their C-termini; TRPCs, TRPVs and TRPMs contain a calmodulin (CaM) binding domain; TRPMLs and TRPPs have a $\mathrm{Ca}^{2+}$-binding EF-hand motif in their C-termini. Most of these domains mediate protein-protein interactions that allow intra- and intercellular associations.

Functionally, TRP channels are activated by diverse stimuli that are physical (e.g., temperature, voltage or mechanical stress) or chemical (e.g., pH, osmotic pressure, neurotransmitters, growth factors, environmental irritants) in nature. This enables TRP channels to act as multifunctional cellular sensors. TRP channels responding to combinations of stimuli serve as polymodal signal detectors for noxious stimuli from a cell's ambient environment (Tominaga et al., 1998; Bautista et 


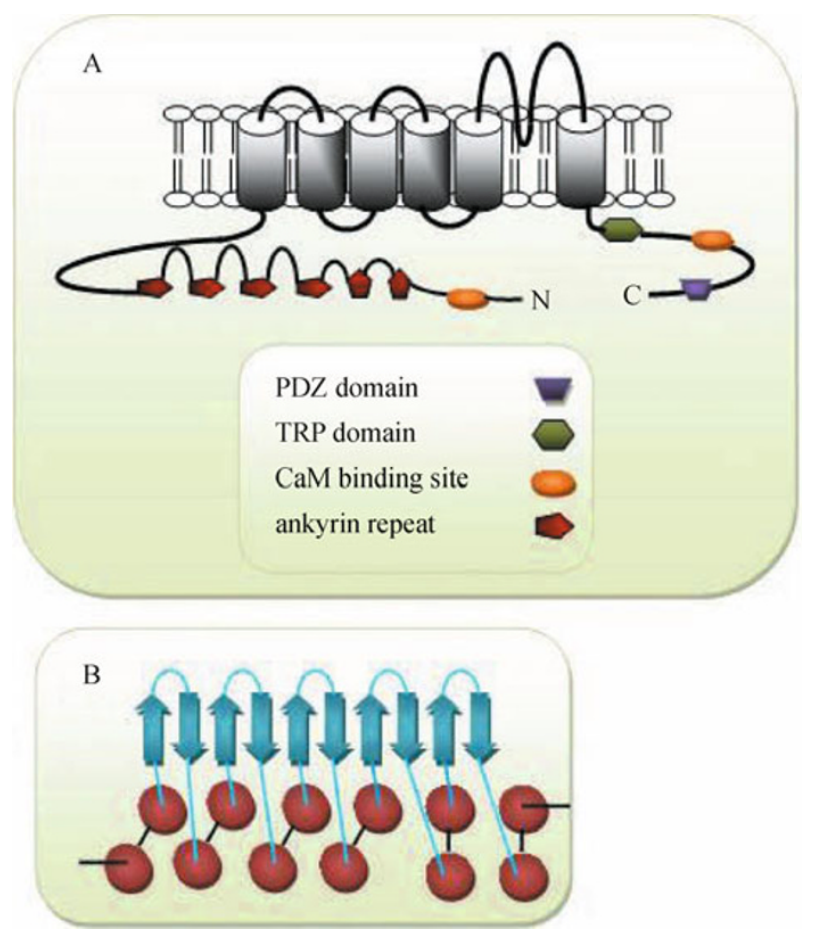

Figure 1. Topological arrangement of a TRPV channel subunit. (A) Each channel subunit contains six transmembrane segments (S1-S6), and a pore region formed by a loop between the S5-S6 segments. Long intracellular $\mathrm{N}$ and $\mathrm{C}$ termini contain several relatively conserved domains such as ankyrin repeat domain (ARD), TRP domain, PDZ domain, CaM binding site. (B) Topological plot of the TRPV ankyrin repeat domain. Red circle represents $\alpha$-helices, and blue arrows and lines represent $\beta$ loop fingers.

al., 2006), or as coincidence detectors for simultaneous signal inputs that contribute to learning and memory (Chuang et al., 2004). As TRP channel subfamilies are defined by sequence homology, channels belonging to the same subfamily may exhibit distinct functional properties. For example, the TRPV subfamily members TRPV1-4 possess exquisite temperature sensitivity important for nociception and thermo-sensing, whereas TRPV5-6 are involved in epithelium $\mathrm{Ca}^{2+}$ entry with ordinary sensitivity to temperature. Similarly, the cold sensor TRPM8 belongs to the TRPM subfamily whose members do not all exhibit high temperature sensitivity.

The physiologic properties of most TRP channels are well studied. Readers are referred to excellent recent reviews on broad TRP channel subjects (Montell, 2005; Owsianik et al., 2006; Nilius, 2007a; Reaves and Wolstenholme, 2007; Gaudet, 2009; Moiseenkova-Bell and Wensel, 2009). In the present review, we focus on studies regarding heteromeric TRP channel formations, their functional diversity, as well as the molecular mechanism governing TRP channel subunits co-assembly.

\section{TRP CHANNELS GENERATED BY SUBUNITS CO-ASSEMBLY}

Subunits heteromerization in TRP channels has been widely reported. Heteromeric channels can form by subunits either within the same subfamily or between different subfamilies (Fig. 2). Compared with other channel families (e.g., the Kv voltage-gated potassium channel family and the ionotropic glutamate receptor family), co-assembly of TRP subunits exhibits substantial specificity.

\section{TRPC}

The TRPC channels are classical or canonical TRPs which are ubiquitously expressed. Based on sequence homology and functional similarities, TRPCs can be further divided into four groups: TRPC1, TRPC2, TRPC3/6/7 and TRPC4/5. Subunit heteromerization in the TRPC subfamily has been extensively explored (Goel et al., 2002; Riccio et al., 2002; Plant and Schaefer, 2003; Schaefer, 2005). A number of independent studies confirmed that co-assembly of TRPC subunits in both native cells and expression systems (Strübing et al., 2001; Goel et al., 2002; Hofmann et al., 2002; Strübing et al., 2003). Intensive interaction between TRPC subunits of three different subgroups (TRPC1, TRPC3/ 6/7 and TRPC4/5) were found (Lintschinger et al., 2000; Liu et al., 2005b; Plant and Schaefer, 2005; Poteser et al., 2006). However, TRPC2, which is thought to be a pseudogene in human, does not interact with any other known TRPC subunit. This is probably due to its distant relationship with other members of the canonical TRP family. Co-assembly of 


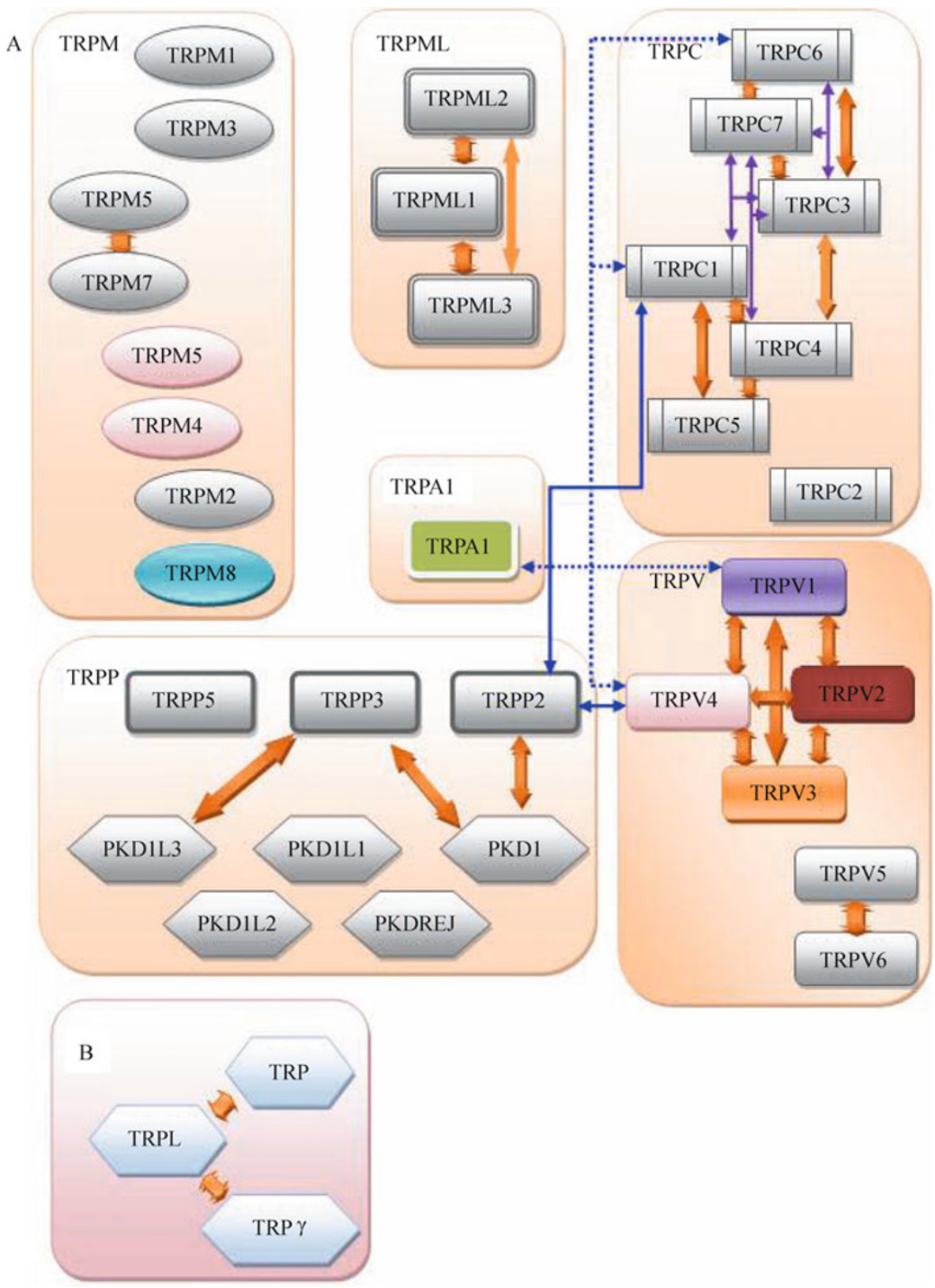

Figure 2. Subunit association between TRP channel subunits. (A) Heteromerization of mammalian TRPs. Orange arrows indicate co-assembly between two channel subunits. Purple arrows indicate interaction among three different subunits of the same subfamily. Blue solid arrows indicate interaction between subunits of different subfamilies. Blue dotted arrows indicate functional interaction for which heteromeric channel has not been identified. (B) Co-assembly of Drosophila TRPs.

TRPC1 and TRPC5 was shown in hippocampal neurons (Strübing et al., 2001; Goel et al., 2002). Similar associations were reported between TRPC1 and TRPC3 via an N-termini domain interaction in salivary gland cells lines (Liu et al., 2005b). In HEK293 cells co-transfection of TRPC1 and TRPC5 (Strübing et al., 2001) or TRPC1 and TRPC4 (Gudermann et al., 2004) yielded novel nonselective cation channels. Zagranichnaya et al. (2005) reported that TRPC1/3/7 can interact to form a store-operated channel complex (Zagranichnaya et al.,
2005). Moreover, heteromerization of TRPC3 and TRPC4 seemed to produce channels with a distinct pore structure clearly different from those of the homomeric TRPC3 and TRPC4 channels (Poteser et al., 2006).

\section{TRPV}

The vanilloid TRPV channels include six members that can be divided into two groups: TRPV1/2/3/4 and TRPV5/6. The 
overall sequence similarity of this subfamily is more than $40 \%$. TRPV1-4 subunits contain 3-6 ankyrin repeats in the intracellular N-terminal region. Activation of TRPV1-4 channels is driven by diverse stimuli including heat, voltage, $\mathrm{pH}$, as well as natural endogenous/extrogenous ligands and synthetic compounds. Indeed, the very noticeable polymodal feature of TRPV1-4 channels indicates fascinating functional implications. As major cellular sensors for thermosensation of dorsal root ganglion (DRG) neurons and epithelial cells, TRPV1-4 channels formed by homomeric subunits exhibit disparate activation temperature thresholds and kinetic properties. TRPV1-4 channels are nonselective cation channels with a permeability ratio for $P_{\mathrm{Ca}} / P_{\mathrm{Na}}$ between one and ten, whereas TRPV5/6 channels are $\mathrm{Ca}^{2+}$-selective cation channels with the $P_{\mathrm{Ca}} / P_{\mathrm{Na}}$ ratio of 100 or more. Several studies revealed that TRPV5/6 subunits not only are colocalized in the same cells but also can form heteromeric channel complexes (Hoenderop et al., 2003; Hellwig et al., 2005; Schaefer, 2005). Co-localization and association between TRPV1 and TRPV2 as well as TRPV1 and TRPV3 were also reported (Smith et al., 2002; Liapi and Wood, 2005; Rutter et al., 2005). Hellwig et al. (2005) reported no association between TRPV1-4 subunits expressed in HEK293 cells except between TRPV1 and TRPV2 subunits (Hellwig et al., 2005). A later study using fluorescence resonance energy transfer (FRET) as well as single-channel recording demonstrated wide-spread interaction between any two members of TRPV1-4 (Cheng et al., 2007). This study provided evidence that thermosensitive TRPV channel subunits can form heteromeric channels with intermediate conductance levels and gating kinetic properties compared to homomeric channels. The disparity between these two studies is attributed to how fluorescence signals were analyzed (Cheng et al., 2007).

\section{TRPP}

Mutations in the polycystic TRPP channels can cause polycystic kidney diseases. The TRPP subfamily contains eight members that can be further divided into two groups by sequence similarities: the PKD1-like group and the PKD2-like group. The PKD1-like group contains five members, PKD1, PKDREJ, PKD1L1, PKD1L2 and PKD1L3; the PKD2-like group contains TRPP2 (previously known as PKD2), TRPP3 (previously known as PKD2L1) and TRPP5 (previously known as PKD2L2). Subunits of the two groups are structurally quite different. PKD1-like group members have 11 transmembrane segments, a very long (up to $~ 3000$ amino acids) extracellular $\mathrm{N}$-terminal segment, and an intracellular C-terminal segment which can interact with that of TRPP2. PKD2-like group members resemble other TRP channels, with intracellular $\mathrm{N}$ and $\mathrm{C}$ termini, six transmembrane segments and a central channel pore region. Members in this group share a coiled-coil domain in their C-termini.
Heteromeric interaction between PKD1 and TRPP2 has been identified by several groups throughout the past decade (Qian et al., 1997; Tsiokas et al., 1997; Hanaoka et al., 2000; Grimm et al., 2003; Delmas et al., 2004; Sharif-Naeini et al., 2009). PKD1 can also interact with PKD2L1 (Murakami et al., 2005); the interaction is essential for PKD2L1 trafficking and channel formation. Along these lines, studies also showed that PKD1L3 and PKD2L1 co-localized in taste receptor cells (Ishimaru et al., 2006; LopezJimenez et al., 2006).

\section{TRPM}

The long or melastatin TRPM subfamily composes of eight members that can be divided into four groups: TRPM1/3, TRPM2/8, TRPM4/5 and TRPM6/7. They are structurally very similar to the well-studied voltage-gated potassium channels, and contain six transmembrane segments, intracellular $\mathrm{N}$ and $C$ termini, and a channel pore domain. Unlike those of TRPV and TRPC subfamilies, TRPM subunits contain a TRP motif with very low sequence conservation but a highly conserved coiled-coil domain within their $\mathrm{C}$ termini. In addition, TRPM channels lack an ankyrin repeat domain seen in the $\mathrm{N}$ terminal of many TRP channels. Furthermore, members of the TRPM3/6/7 group have a functional enzymatic domain in their $\mathrm{C}$ termini. Most TRPMs except TRPM4/5 (TRPM4 and TRPM5 are the only monovalent-selective ion channel of the TRP family) are cation channels permeable to $\mathrm{Ca}^{2+}$ and $\mathrm{Mg}^{2+}$. All TRPM subunits can form functional homotetrameric channels. Whether TRPM subunits can heteromerize remains far less clear. The only known combination is between TRPM6 and TRPM7 (Chubanov et al., 2004; Chubanov et al., 2005; Li et al., 2006; Jiang, 2007), in which case heteromeric channels with intermediate conductance and gating properties exhibit distinct properties compared to the parental homomers, such as different sensitivity to low $\mathrm{PH}$, different permeability to divalent cation and different regulation by 2-APB (Li et al., 2006).

\section{TRPML}

The mucolipin TRPML family exhibited high homology with TRPPs but low similarity to other TRPs. This family of nonselective cation channels consists of three members, referred to as TRPML1, TRPML2, and TRPML3. Mutation of human TRPML1 can cause mucolipidosis type IV, a neurodegenerative lysosomal storage disorder disease (Bargal et al., 2000). Mutation of TRPML3 in varitint-waddler mouse resulted in deafness and pigmentation defects ( $\mathrm{Di}$ Palma et al., 2002). Using a FRET-based approach Montell and coworkers demonstrated that TRPMLs can interact to form heteromultimers (Venkatachalam et al., 2006). Moreover, the presence of either TRPML1 or TRPML2 specifically make TRPML3 trafficking to lysosomes from endoplasmic reticulum (Venkatachalam et al., 2006). Recent study which 
using immunocytochemical analysis as well as singlechannel recording confirmed that heteromultimeric TRPMLs exhibit intermediate conductance and kinetic properties (Curcio-Morelli et al., 2010).

\section{Heteromerization between TRP subunits of different subfamilies}

Since the early reports of TRP subunits heteromerization in Drosophila (Gillo et al., 1996; Xu et al., 1997), there are extensive documentations appeared in the past decade on TRP channel subunits co-assembly into heteromeric complex. Studies continuous to reveal wide-spread heteromerization within the TRP channel superfamily. TRPY (a Drosophila TRP-related subunit) can co-assemble with TRPL to form a channel stimulated by phospholipase C(PLC) (Xu et al., 2000). Independent studies from several groups focusing on TRPC1 and TRPP2 have demonstrated that heteromeric channels formed by these two subunit types at a 2:2 stoichiometry exhibited a new receptor-operated channel property (Tsiokas et al., 1999; Bai et al., 2008; Kobori et al., 2009; Zhang et al., 2009). Köttgen et al. (2008) reported that TRPV4 and TRPP2 formed heteromeric channel complex both in vivo and in vitro (Köttgen et al., 2009). Then using atomic force microscopy, it was found that TRPP2 and TRPV4 form heteromeric complexes with a 2:2 stoichiometry and alternating subunit arrangement (Stewart et al., 2010). Moreover, novel combinations including TRPC1/TRPC6/ TRPV4 and TRPA1/TRPV1 have been reported by recent studies (Alessandri-Haber et al., 2009; Salas et al., 2009).

\section{MOLECULAR MECHANISMS GOVERNING TRP CHANNEL SUBUNITS ASSEMBLY}

What are the molecular mechanisms governing TRP channel assembly? Recent studies have applied a combination of biochemistry, electrophysiology, X-ray crystallography as well as single-molecule optical imaging to elucidate the answer. These studies have revealed a number of molecular determinants (Lepage and Boulay, 2007; Schindl and Romanin, 2007) that could directly or indirectly contribute to TRP channel subunits assembly.

\section{Ankyrin repeat domain}

Ankyrin repeat domain (ARD) is a repetition of 33-residue motif. Each ankyrin repeat is characterized by a pair of antiparallel $\alpha$-helices followed by a loop linking to a $\beta$-hairpin (Sedgwick and Smerdon, 1999). Generally, most proteins contain tandem arrays of two-to-seven repeats (Groves and Barford, 1999). ARD is one of the most common domains mediating protein-protein interaction. For the majority of cases, ARD interacts with a short peptide segment; ARD$A R D$ interactions are rarely observed. In TRP channels, the
$A R D$ resides in the intracellular $N$ terminus. The number of repeats varies from 3-6 for TRPC/TRPV, 14-15 for TRPA and $\sim 29$ for TRPN. TRPM, TRPP, and TRPML channels lack the $A R D$. It has been reported that ARD plays a key role in heteromerization of TRPC $1 / 3$, and the first ankyrin repeat of TRPC1 is found to interact with the $\mathrm{N}$ terminus of TRPC3 (Liu et al., 2005b). Other studies demonstrated that not only Nterminal ankyrin repeat domain (Hellwig et al., 2005; Arniges et al., 2006) but also the C-terminal domain from position 716 to position 828 participates in oligomerization and trafficking of TRPV4 (Hellwig et al., 2005; Arniges et al., 2006; Becker et al., 2008). Meanwhile, Lepage et al. (2006) found that both $\mathrm{N}$ terminal ARD and $C$ terminus are responsible for TRPC oligomerization (Lepage et al., 2006). Two studies reported that ARD is critical for channel assembly of TRPV5 and TRPV6 (Chang et al., 2004; Erler et al., 2004). Specifically, the first ankyrin repeat (residues 64-77) of TRPV5 and the third ankyrin repeat (residues 116-140) of TRPV6 are important for subunit assembly. However, these studies did not exclude other factors which may regulate channel assembly. A recent study using analytical size exclusion chromatography as well as crystallization provided evidence that TRPV6-ARD is monomeric in solution, does not form tetrameric complexes, and may regulate channel assembly partly (Phelps et al., 2008). Furthermore, for TRPV2, both solution studies and crystal packing interactions revealed that the ankyrin repeat domain is a regulatory domain but not a determinant of subunit assembly (Jin et al., 2006; McCleverty et al., 2006).

\section{Coiled-coil domain}

Coiled-coil domain is a structural motif that consists of two or more $\alpha$-helices that wrap together like the strands of a rope. They are one of the most common and best understood protein-protein interaction domains. Usually it contains a heptad repeat pattern of (abcdefg $)_{n}$ in its amino acid sequence. The first and fourth positions of the heptad repeat are occupied by hydrophobic amino acids while the fifth and seventh position residues are charged or polar. Coiled-coil domain has been found in members of TRPC, TRPM and TRPP subfamilies. It has been reported that the $\mathrm{N}$-terminal coiled-coil structure domain of TRPC1 facilitated the homodimerization process (Engelke et al., 2002). Interesting studies with TRPM2 and TRPM8 demonstrated that the coiled-coil domain in the intracellular $C$ terminus instead of the $\mathrm{N}$ terminus is essential for channel tetramerization (Mei et al., 2006; Tsuruda et al., 2006; Mei and Jiang, 2009). The Cterminal coiled-coil domain of TRPP2 has been demonstrated to be essential for interaction with the C-terminal part of PKD1 (Hanaoka et al., 2000; Delmas, 2005). A recent study provides evidence that the C-terminal coiled-coil domain directs assembly of homotrimer of TRPP2, which in turn interacts with C-termini of PKD1 (Yu et al., 2009). The case is 
very similar to the rod cyclic nucleotide-gated channels in which the C-terminal coiled-coil domain dedicates the assembly of three CNGA1 subunits and one CNGB1 subunit (Zhong et al., 2002).

\section{TRP domain}

The TRP domain is a 25-amino acid motif rich in charged residues. It contains the TRP box (characterized by the specific amino acid sequence EWKFAR), which is fully conserved in TRPC channels, but is less conserved among members of the TRPV and TRPM subfamilies. The TRP domain is localized in the $C$ terminus close to the sixth transmembrane segment (Clapham et al., 2001). Studies indicated that the TRP domain may be essential for TRP channel assembly. It has been recently shown that the TRP domain of TRPV1, in particular the segment from D684 to R721, is involved in assembly of the channel subunits into functional channels (Garciasanz et al., 2004). The TRP domain may also serve as a regulatory domain targeted by PIP2 and other channel regulators (Liu and Liman, 2003; Liu et al., 2005a; Rohács et al., 2005).

\section{Other factors}

Effects to identify the determinants of TRP subunits assembly also led to other regions. Boulay and coworkers identified two regions in TRPC that are involved in subunits assembly (Lepage et al., 2006). One region is in the $\mathrm{N}$-terminal region that includes the ankyrin repeat domain and the coiled-coil domain; the other region is made of the pore region and the Cterminal tail (Lepage et al., 2006). Studies suggested that both the $\mathrm{C}$-terminal and the $\mathrm{N}$-terminal may be necessary for tetrameric assembly of TRPP2,TRPV4, and TRPV5 (Chang et al., 2004; Becker et al., 2008; Feng et al., 2008; Yu et al., 2009). For TRPV5, Chang et al. (2004) narrowed down the essential sequence to parts of the $\mathrm{N}$ terminus (residues 64-77) and the $C$ terminus (residues 596-601) (Chang et al., 2004). Previous studies also identified the transmembrane domain as a candidate for the molecular determinants (Xu et al., 1997, 2000; Hellwig et al., 2005). The PDZ domain of TRPC4, which controls the channel's cellular localization and surface expression in HEK293 cells, was suggested to be a potential determinant of channel assembly as well (Mery et al., 2002). Additional efforts are needed to carefully examine the identified candidate structures and identify potential new structures that may contribute to TRP channel assembly.

\section{HETEROMERIZATION EXTENDS THE FUNCTIONAL DIVERSITY OF TRP CHANNELS}

While much remains to be learned about the physiologic consequences of heteromultimerization among TRP subunits, it is clear that subunits co-assembly yields a variety of channel types with functional properties distinct from their homomeric counterparts. In the early study of Drosophila heteromeric TRP channels, it was shown that the combination of TRP/TRPL subunits can produce a distinct store-operated conductance ( $\mathrm{Xu}$ et al., 1997). In addition, co-assembly of TRPL and TRPy yielded a channel that was activated by PLC stimulation (Xu et al., 2000). Meanwhile, heteromerization of mammalian TRPV1/3, TRPV5/6, TRPML1/2, TRPML1/3, TRPML2/3,TRPC1/4, TRPC1/5, TRPC3/4, TRPC3/6, TRPC $3 / 7$ or TRPC $4 / 5$ has been identified to produce channels with novel properties (Lintschinger et al., 2000; Strübing et al., 2001; Hoenderop et al., 2003; Strübing et al., 2003; Plant and Schaefer, 2005; Poteser et al., 2006; Cheng et al., 2007; Curcio-Morelli et al., 2010). TRPV 3 was found to co-assemble with TRPV1 to form heteromeric channels with altered pharmacological properties (Smith et al., 2002). Heteromultimeric TRPP2/TRPC1 channels have been identified to be new receptor-operated channels implicated in mechanosensation (Tsiokas et al., 1999; Bai et al., 2008; Kobori et al., 2009). Furthermore, TRPP2 associates with TRPV4 to produce a channel that functions as a mechanosensitive and thermosensitive molecular sensor in the primary cilium of renal epithelium cells (Köttgen et al., 2008). TRPC1/3/7 can interact to produce a channel complex with store-operated channel properties (Zagranichnaya et al., 2005). Alessandri-Haber et al. (2009) found that TRPC1 and TRPC6 with TRPV4 are frequently co-expressed in DRG neurons; TRPC1 and TRPC6 subunits incorporate with TRPV4 to mediate mechanical hyperalgesia and primary afferent nociceptor sensitization (Alessandri-Haber et al., 2009). Surprisingly, the noxious cold-sensitive TRPA1 channel subunit may functionally interact with the noxious heatsensitive TRPV1 channel subunit, despite their substantial sequence dissimilarity. It is found that co-expression of TRPA1 and TRPV1 contributes to TRPA1-mediated responses in trigeminal sensory neurons (Salas et al., 2009). Moreover, PKD1L3 was found co-localized with PKD2L1 in taste receptor cells (Ishimaru et al., 2006; LopezJimenez et al., 2006) and may play a potential role in taste sensory transduction. Taken together, heteromerization of TRPs serves as an important mechanism for the regulation of TRP channel function.

While most studies so far focus on the static TRP channels subunit composition, what is perhaps more interesting is the possibility that the subunit composition of TRP channels in living cells may be dynamically regulated. Indeed, upon stimulation the cell surface TRP channel density increases dramatically and rapidly (Vetter et al., 2008; Schmidt et al., 2009). Little is known about whether and how TRP channels change subunit composition in response to environmental stimuli.

\section{CONCLUSION}

TRP channels are widely expressed in virtually all cell types 
and carry out diverse fundamental functions in the human body. Heteromerization of TRP subunits emerges as one way to produce diverse functions of this fascinating family. This is the area that still needs much work. This review provides an overview of the current understanding of TRP channel assembly and function. Further studies will fully reveal the scope of subunits co-assembly and the molecular mechanism governing specific subunits interactions.

\section{REFERENCES}

Alessandri-Haber, N., Dina, O.A., Chen, X., and Levine, J.D. (2009). TRPC1 and TRPC6 channels cooperate with TRPV4 to mediate mechanical hyperalgesia and nociceptor sensitization. J Neurosci 29, 6217-6228.

Arniges, M., Fernández-Fernández, J.M., Albrecht, N., Schaefer, M., and Valverde, M.A. (2006). Human TRPV4 channel splice variants revealed a key role of ankyrin domains in multimerization and trafficking. J Biol Chem 281, 1580-1586.

Bai, C.X., Giamarchi, A., Rodat-Despoix, L., Padilla, F., Downs, T., Tsiokas, L., and Delmas, P. (2008). Formation of a new receptoroperated channel by heteromeric assembly of TRPP2 and TRPC1 subunits. EMBO Rep 9, 472-479.

Bargal, R., Avidan, N., Ben-Asher, E., Olender, Z., Zeigler, M., Frumkin, A., Raas-Rothschild, A., Glusman, G., Lancet, D., and Bach, G. (2000). Identification of the gene causing mucolipidosis type IV. Nat Genet 26, 118-123.

Bautista, D.M., Jordt, S.E., Nikai, T., Tsuruda, P.R., Read, A.J., Poblete, J., Yamoah, E.N., Basbaum, A.I., and Julius, D. (2006). TRPA1 mediates the inflammatory actions of environmental irritants and proalgesic agents. Cell 124, 1269-1282.

Becker, D., Müller, M., Leuner, K., and Jendrach, M. (2008). The Cterminal domain of TRPV4 is essential for plasma membrane localization. Mol Membr Biol 25, 139-151.

Chang, Q., Gyftogianni, E., van de Graaf, S.F., Hoefs, S., Weidema, F. A., Bindels, R.J., and Hoenderop, J.G. (2004). Molecular determinants in TRPV5 channel assembly. J Biol Chem 279, 54304-54311.

Cheng, W., Yang, F., Takanishi, C.L., and Zheng, J. (2007). Thermosensitive TRPV channel subunits coassemble into heteromeric channels with intermediate conductance and gating properties. J Gen Physiol 129, 191-207.

Chuang, H.H., Neuhausser, W.M., and Julius, D. (2004). The supercooling agent icilin reveals a mechanism of coincidence detection by a temperature-sensitive TRP channel. Neuron 43, 859-869.

Chubanov, V., Mederos y Schnitzler, M., Wäring, J., Plank, A., and Gudermann, T. (2005). Emerging roles of TRPM6/TRPM7 channel kinase signal transduction complexes. Naunyn Schmiedebergs Arch Pharmacol 371, 334-341.

Chubanov, V., Waldegger, S., Mederos y Schnitzler, M., Vitzthum, H., Sassen, M.C., Seyberth, H.W., Konrad, M., and Gudermann, T. (2004). Disruption of TRPM6/TRPM7 complex formation by a mutation in the TRPM6 gene causes hypomagnesemia with secondary hypocalcemia. Proc Natl Acad Sci U S A 101, 2894-2899.

Clapham, D.E. (2003). TRP channels as cellular sensors. Nature 426, 517-524.

Clapham, D.E., Runnels, L.W., and Strübing, C. (2001). The TRP ion channel family. Nat Rev Neurosci 2, 387-396.

Cosens, D.J., and Manning, A. (1969). Abnormal electroretinogram from a Drosophila mutant. Nature 224, 285-287.

Curcio-Morelli, C., Zhang, P., Venugopal, B., Charles, F.A., Browning, M.F., Cantiello, H.F., and Slaugenhaupt, S.A. (2010). Functional multimerization of mucolipin channel proteins. J Cell Physiol 222, 328-335.

Delmas, P. (2005). Polycystins: polymodal receptor/ion-channel cellular sensors. Pflugers Arch 451, 264-276.

Delmas, P., Nauli, S.M., Li, X., Coste, B., Osorio, N., Crest, M., Brown, D.A., and Zhou, J. (2004). Gating of the polycystin ion channel signaling complex in neurons and kidney cells. FASEB $\mathrm{J} 18$, 740-742.

Di Palma, F., Belyantseva, I.A., Kim, H.J., Vogt, T.F., Kachar, B., and Noben-Trauth, K. (2002). Mutations in Mcoln3 associated with deafness and pigmentation defects in varitint-waddler (Va) mice. Proc Natl Acad Sci U S A 99, 14994-14999.

Engelke, M., Friedrich, O., Budde, P., Schäfer, C., Niemann, U., Zitt, C., Jüngling, E., Rocks, O., Lückhoff, A., and Frey, J. (2002). Structural domains required for channel function of the mouse transient receptor potential protein homologue TRP1beta. FEBS Lett 523, 193-199.

Erler, I., Hirnet, D., Wissenbach, U., Flockerzi, V., and Niemeyer, B.A. (2004). Ca2+-selective transient receptor potential V channel architecture and function require a specific ankyrin repeat. J Biol Chem 279, 34456-34463.

Feng, S., Okenka, G.M., Bai, C.X., Streets, A.J., Newby, L.J., DeChant, B.T., Tsiokas, L., Obara, T., and Ong, A.C. (2008). Identification and functional characterization of an N-terminal oligomerization domain for polycystin-2. J Biol Chem 283, 28471-28479.

García-Sanz, N., Fernández-Carvajal, A., Morenilla-Palao, C., Planells-Cases, R., Fajardo-Sánchez, E., Fernández-Ballester, G., and Ferrer-Montiel, A. (2004). Identification of a tetramerization domain in the $\mathrm{C}$ terminus of the vanilloid receptor. J Neurosci 24 , 5307-5314.

Gaudet, R. (2009). Divide and conquer: high resolution structural information on TRP channel fragments. J Gen Physiol 133, 231-237.

Gillo, B., Chorna, I., Cohen, H., Cook, B., Manistersky, I., Chorev, M., Arnon, A., Pollock, J.A., Selinger, Z., and Minke, B. (1996). Coexpression of Drosophila TRP and TRP-like proteins in Xenopus oocytes reconstitutes capacitative $\mathrm{Ca} 2+$ entry. Proc Natl Acad Sci U S A 93, 14146-14151.

Goel, M., Sinkins, W.G., and Schilling, W.P. (2002). Selective association of TRPC channel subunits in rat brain synaptosomes. J Biol Chem 277, 48303-48310.

Grimm, D.H., Cai, Y., Chauvet, V., Rajendran, V., Zeltner, R., Geng, L., Avner, E.D., Sweeney, W., Somlo, S., and Caplan, M.J. (2003). Polycystin-1 distribution is modulated by polycystin-2 expression in mammalian cells. J Biol Chem 278, 36786-36793.

Groves, M.R., and Barford, D. (1999). Topological characteristics of helical repeat proteins. Curr Opin Struct Biol 9, 383-389.

Gudermann, T., Hofmann, T., Mederos, Y.S.M., and Dietrich, A. (2004). Activation, subunit composition and physiological relevance of DAG-sensitive TRPC proteins. Novartis Found Symp 258, 103-118; discussion 118-122, 155-109, 263-106.

Hanaoka, K., Qian, F., Boletta, A., Bhunia, A.K., Piontek, K., Tsiokas, 
L., Sukhatme, V.P., Guggino, W.B., and Germino, G.G. (2000). Coassembly of polycystin-1 and -2 produces unique cation-permeable currents. Nature 408, 990-994.

Hellwig, N., Albrecht, N., Harteneck, C., Schultz, G., and Schaefer, M. (2005). Homo- and heteromeric assembly of TRPV channel subunits. J Cell Sci 118, 917-928.

Hoenderop, J.G., Voets, T., Hoefs, S., Weidema, F., Prenen, J., Nilius, B., and Bindels, R.J. (2003). Homo- and heterotetrameric architecture of the epithelial $\mathrm{Ca}^{2+}$ channels TRPV5 and TRPV6. EMBO J 22, 776-785.

Hofmann, T., Schaefer, M., Schultz, G., and Gudermann, T. (2002). Subunit composition of mammalian transient receptor potential channels in living cells. Proc Natl Acad Sci U S A 99, 74617466.

Ishimaru, Y., Inada, H., Kubota, M., Zhuang, H., Tominaga, M., and Matsunami, H. (2006). Transient receptor potential family members PKD1L3 and PKD2L1 form a candidate sour taste receptor. Proc Natl Acad Sci U S A 103, 12569-12574.

Jiang, L.H. (2007). Subunit interaction in channel assembly and functional regulation of transient receptor potential melastatin (TRPM) channels. Biochem Soc Trans 35, 86-88.

Jin, X., Touhey, J., and Gaudet, R. (2006). Structure of the N-terminal ankyrin repeat domain of the TRPV2 ion channel. J Biol Chem 281, 25006-25010.

Kobori, T., Smith, G.D., Sandford, R., and Edwardson, J.M. (2009). The transient receptor potential channels TRPP2 and TRPC1 form a heterotetramer with a 22 stoichiometry and an alternating subunit arrangement. J Biol Chem 284, 35507-35513.

Köttgen, M., Buchholz, B., Garcia-Gonzalez, M.A., Kotsis, F., Fu, X., Doerken, M., Boehlke, C., Steffl, D., Tauber, R., Wegierski, T., et al. (2008). TRPP2 and TRPV4 form a polymodal sensory channel complex. J Cell Biol 182, 437-447.

Lepage, P.K., and Boulay, G. (2007). Molecular determinants of TRP channel assembly. Biochem Soc Trans 35, 81-83.

Lepage, P.K., Lussier, M.P., Barajas-Martinez, H., Bousquet, S.M., Blanchard, A.P., Francoeur, N., Dumaine, R., and Boulay, G. (2006). Identification of two domains involved in the assembly of transient receptor potential canonical channels. J Biol Chem 281, 30356-30364.

Li, M., Jiang, J., and Yue, L. (2006). Functional characterization of homo- and heteromeric channel kinases TRPM6 and TRPM7. J Gen Physiol 127, 525-537.

Liapi, A., and Wood, J.N. (2005). Extensive co-localization and heteromultimer formation of the vanilloid receptor-like protein TRPV2 and the capsaicin receptor TRPV1 in the adult rat cerebral cortex. Eur J Neurosci 22, 825-834.

Lintschinger, B., Balzer-Geldsetzer, M., Baskaran, T., Graier, W.F., Romanin, C., Zhu, M.X., and Groschner, K. (2000). Coassembly of Trp1 and Trp3 proteins generates diacylglycerol- and $\mathrm{Ca}^{2+}$ sensitive cation channels. J Biol Chem 275, 27799-27805.

Liu, B., Zhang, C., and Qin, F. (2005a). Functional recovery from desensitization of vanilloid receptor TRPV1 requires resynthesis of phosphatidylinositol 4,5-bisphosphate. J Neurosci 25, 4835- 4843.

Liu, D., and Liman, E.R. (2003). Intracellular $\mathrm{Ca}^{2+}$ and the phospholipid PIP2 regulate the taste transduction ion channel TRPM5. Proc Natl Acad Sci U S A 100, 15160-15165.

Liu, X., Bandyopadhyay, B.C., Singh, B.B., Groschner, K., and Ambudkar, I.S. (2005b). Molecular analysis of a store-operated and 2-acetyl-sn-glycerol-sensitive non-selective cation channel. Heteromeric assembly of TRPC1-TRPC3. J Biol Chem 280, 21600-21606.

LopezJimenez, N.D., Cavenagh, M.M., Sainz, E., Cruz-Ithier, M.A., Battey, J.F., and Sullivan, S.L. (2006). Two members of the TRPP family of ion channels, Pkd1/3 and Pkd2l1, are co-expressed in a subset of taste receptor cells. J Neurochem 98, 68-77.

McCleverty, C.J., Koesema, E., Patapoutian, A., Lesley, S.A., and Kreusch, A. (2006). Crystal structure of the human TRPV2 channel ankyrin repeat domain. Protein Sci 15, 2201-2206.

Mei, Z.Z., and Jiang, L.H. (2009). Requirement for the N-terminal coiled-coil domain for expression and function, but not subunit interaction of, the ADPR-activated TRPM2 channel. J Membr Biol 230, 93-99.

Mei, Z.Z., Xia, R., Beech, D.J., and Jiang, L.H. (2006). Intracellular coiled-coil domain engaged in subunit interaction and assembly of melastatin-related transient receptor potential channel 2. J Biol Chem 281, 38748-38756.

Mery, L., Strauss, B., Dufour, J.F., Krause, K.H., and Hoth, M. (2002). The PDZ-interacting domain of TRPC4 controls its localization and surface expression in HEK293 cells. J Cell Sci 115, 3497-3508.

Moiseenkova-Bell, V.Y., and Wensel, T.G. (2009). Hot on the trail of TRP channel structure. J Gen Physiol 133, 239-244.

Montell, C. (2005). The TRP superfamily of cation channels. Sci STKE 2005, re3.

Murakami, M., Ohba, T., Xu, F., Shida, S., Satoh, E., Ono, K., Miyoshi, I., Watanabe, H., Ito, H., and lijima, T. (2005). Genomic organization and functional analysis of murine PKD2L1. J Biol Chem 280, 5626-5635.

Nilius, B. (2007a). Transient receptor potential (TRP) cation channels: rewarding unique proteins. Bull Mem Acad R Med Belg 162, 244-253.

Nilius, B. (2007b). TRP channels in disease. Biochim Biophys Acta 1772, 805-812.

Owsianik, G., D'hoedt, D., Voets, T., and Nilius, B. (2006). Structurefunction relationship of the TRP channel superfamily. Rev Physiol Biochem Pharmacol 156, 61-90.

Phelps, C.B., Huang, R.J., Lishko, P.V., Wang, R.R., and Gaudet, R. (2008). Structural analyses of the ankyrin repeat domain of TRPV6 and related TRPV ion channels. Biochemistry 47, 2476-2484.

Plant, T.D., and Schaefer, M. (2003). TRPC4 and TRPC5: receptoroperated $\mathrm{Ca}^{2+}$-permeable nonselective cation channels. Cell Calcium 33, 441-450.

Plant, T.D., and Schaefer, M. (2005). Receptor-operated cation channels formed by TRPC4 and TRPC5. Naunyn Schmiedebergs Arch Pharmacol 371, 266-276.

Poteser, M., Graziani, A., Rosker, C., Eder, P., Derler, I., Kahr, H., Zhu, M.X., Romanin, C., and Groschner, K. (2006). TRPC3 and TRPC4 associate to form a redox-sensitive cation channel. Evidence for expression of native TRPC3-TRPC4 heteromeric channels in endothelial cells. J Biol Chem 281, 13588-13595.

Qian, F., Germino, F.J., Cai, Y., Zhang, X., Somlo, S., and Germino, G. G. (1997). PKD1 interacts with PKD2 through a probable coiledcoil domain. Nat Genet 16, 179-183.

Reaves, B.J., and Wolstenholme, A.J. (2007). The TRP channel superfamily: insights into how structure, protein-lipid interactions and localization influence function. Biochem Soc Trans 35, 77-80.

Riccio, A., Medhurst, A.D., Mattei, C., Kelsell, R.E., Calver, A.R., 
Randall, A.D., Benham, C.D., and Pangalos, M.N. (2002). mRNA distribution analysis of human TRPC family in CNS and peripheral tissues. Brain Res Mol Brain Res 109, 95-104.

Rohács, T., Lopes, C.M., Michailidis, I., and Logothetis, D.E. (2005). $\mathrm{PI}(4,5) \mathrm{P} 2$ regulates the activation and desensitization of TRPM8 channels through the TRP domain. Nat Neurosci 8, 626-634.

Rutter, A.R., Ma, Q.P., Leveridge, M., and Bonnert, T.P. (2005). Heteromerization and colocalization of TrpV1 and TrpV2 in mammalian cell lines and rat dorsal root ganglia. Neuroreport 16, 1735-1739.

Salas, M.M., Hargreaves, K.M., and Akopian, A.N. (2009). TRPA1mediated responses in trigeminal sensory neurons: interaction between TRPA1 and TRPV1. Eur J Neurosci 29, 1568-1578.

Schaefer, M. (2005). Homo- and heteromeric assembly of TRP channel subunits. Pflugers Arch 451, 35- 42.

Schindl, R., and Romanin, C. (2007). Assembly domains in TRP channels. Biochem Soc Trans 35, 84-85.

Schmidt, M., Dubin, A.E., Petrus, M.J., Earley, T.J., and Patapoutian, A. (2009). Nociceptive signals induce trafficking of TRPA1 to the plasma membrane. Neuron 64, 498-509.

Sedgwick, S.G., and Smerdon, S.J. (1999). The ankyrin repeat: a diversity of interactions on a common structural framework. Trends Biochem Sci 24, 311-316.

Sharif-Naeini, R., Folgering, J.H., Bichet, D., Duprat, F., Lauritzen, I., Arhatte, M., Jodar, M., Dedman, A., Chatelain, F.C., Schulte, U., et al. (2009). Polycystin-1 and -2 dosage regulates pressure sensing. Cell 139, 587-596.

Smith, G.D., Gunthorpe, M.J., Kelsell, R.E., Hayes, P.D., Reilly, P., Facer, P., Wright, J.E., Jerman, J.C., Walhin, J.P., Ooi, L., et al. (2002). TRPV3 is a temperature-sensitive vanilloid receptor-like protein. Nature 418, 186-190.

Stewart, A.P., Smith, G.D., Sandford, R.N., and Edwardson, J.M. (2010). Atomic force microscopy reveals the alternating subunit arrangement of the TRPP2-TRPV4 heterotetramer. Biophys J 99, 790-797.

Stowers, L., Holy, T.E., Meister, M., Dulac, C., and Koentges, G. (2002). Loss of sex discrimination and male-male aggression in mice deficient for TRP2. Science 295, 1493-1500.

Strübing, C., Krapivinsky, G., Krapivinsky, L., and Clapham, D.E. (2001). TRPC1 and TRPC5 form a novel cation channel in mammalian brain. Neuron 29, 645-655.

Strübing, C., Krapivinsky, G., Krapivinsky, L., and Clapham, D.E. (2003). Formation of novel TRPC channels by complex subunit interactions in embryonic brain. J Biol Chem 278, 39014-39019.

Tominaga, M., Caterina, M.J., Malmberg, A.B., Rosen, T.A., Gilbert, H., Skinner, K., Raumann, B.E., Basbaum, A.I., and Julius, D. (1998). The cloned capsaicin receptor integrates multiple pain- producing stimuli. Neuron 21, 531-543.

Tsiokas, L., Arnould, T., Zhu, C., Kim, E., Walz, G., and Sukhatme, V. P. (1999). Specific association of the gene product of PKD2 with the TRPC1 channel. Proc Natl Acad Sci U S A 96, 3934-3939.

Tsiokas, L., Kim, E., Arnould, T., Sukhatme, V.P., and Walz, G. (1997). Homo- and heterodimeric interactions between the gene products of PKD1 and PKD2. Proc Natl Acad Sci U S A 94, 6965-6970.

Tsuruda, P.R., Julius, D., and Minor, D.L. Jr. (2006). Coiled coils direct assembly of a cold-activated TRP channel. Neuron 51, 201-212.

Venkatachalam, K., Hofmann, T., and Montell, C. (2006). Lysosomal localization of TRPML3 depends on TRPML2 and the mucolipidosis-associated protein TRPML1. J Biol Chem 281, 17517-17527.

Vetter, I., Cheng, W., Peiris, M., Wyse, B.D., Roberts-Thomson, S.J., Zheng, J., Monteith, G.R., and Cabot, P.J. (2008). Rapid, opioidsensitive mechanisms involved in transient receptor potential vanilloid 1 sensitization. J Biol Chem 283, 19540-19550.

Xu, X.Z., Chien, F., Butler, A., Salkoff, L., and Montell, C. (2000). TRPgamma, a drosophila TRP-related subunit, forms a regulated cation channel with TRPL. Neuron 26, 647-657.

Xu, X.Z., Li, H.S., Guggino, W.B., and Montell, C. (1997). Coassembly of TRP and TRPL produces a distinct store-operated conductance. Cell 89, 1155-1164.

Yu, Y., Ulbrich, M.H., Li, M.H., Buraei, Z., Chen, X.Z., Ong, A.C., Tong, L., Isacoff, E.Y., and Yang, J. (2009). Structural and molecular basis of the assembly of the TRPP2/PKD1 complex. Proc Natl Acad Sci U S A 106, 11558-11563.

Zagranichnaya, T.K., Wu, X., and Villereal, M.L. (2005). Endogenous TRPC1, TRPC3, and TRPC7 proteins combine to form native store-operated channels in HEK-293 cells. J Biol Chem 280, 29559-29569.

Zhang, P., Luo, Y., Chasan, B., González-Perrett, S., Montalbetti, N., Timpanaro, G.A., Cantero, M.R., Ramos, A.J., Goldmann, W.H., Zhou, J., et al. (2009). The multimeric structure of polycystin-2 (TRPP2): structural-functional correlates of homo- and heteromultimers with TRPC1. Hum Mol Genet 18, 1238-1251.

Zhang, Y., Hoon, M.A., Chandrashekar, J., Mueller, K.L., Cook, B., Wu, D., Zuker, C.S., and Ryba, N.J. (2003). Coding of sweet, bitter, and umami tastes: different receptor cells sharing similar signaling pathways. Cell 112, 293-301.

Zhong, H., Molday, L.L., Molday, R.S., and Yau, K.W. (2002). The heteromeric cyclic nucleotide-gated channel adopts a $3 \mathrm{~A}: 1 \mathrm{~B}$ stoichiometry. Nature 420, 193-198.

Zhou, X.L., Batiza, A.F., Loukin, S.H., Palmer, C.P., Kung, C., and Saimi, Y. (2003). The transient receptor potential channel on the yeast vacuole is mechanosensitive. Proc Natl Acad Sci U S A 100, 7105-7110. 